\title{
The spectrum of endometrial pathology induced by progesterone receptor modulators
}

\author{
George L Mutter ${ }^{1}$, Christine Bergeron ${ }^{2}$, Liane Deligdisch $^{3}$, Alex Ferenczy ${ }^{4}$, Mick Glant ${ }^{5}$, \\ Maria Merino ${ }^{6}$, Alistair RW Williams ${ }^{7}$ and Diana L Blithe ${ }^{8}$ \\ ${ }^{1}$ Department of Pathology, Brigham and Women's Hospital, Boston, MA, USA; ${ }^{2}$ Department of Pathology, \\ Laboratoire Pasteur-Cerba, Cergy Pontoise, France; ${ }^{3}$ Department of Pathology, Mount Sinai School of \\ Medicine, New York, NY, USA; ${ }^{4}$ Department of Pathology, Jewish General Hospital, Montreal, QC, Canada; \\ ${ }^{5}$ Department of Pathology, DCL Medical Laboratories Inc., Indianapolis, IN, USA; ${ }^{6}$ Department of Pathology, \\ National Institutes of Health, Bethesda, MD, USA; ${ }^{7}$ Department of Pathology, University of Edinburgh, \\ Edinburgh, UK and ${ }^{8}$ The Contraception and Reproductive Health Branch, Center for Population Research, \\ National Institute of Child Health and Human Development, Bethesda, MD, USA
}

\begin{abstract}
Progesterone receptor modulators (PRM) are hormonally active drugs effective in the management of endometriosis and uterine leiomyomata. The endometrial effects of progestin blockade by PRMs in premenopausal women are currently being evaluated in several clinical trials, but few pathologists have had access to these materials and published information of the histological changes is scanty. Eighty-four endometrial specimens from women receiving one of four different PRMs were reviewed by a panel of seven experienced gynecologic pathologists to develop consensus observations and interpretive recommendations as part of an NIH-sponsored workshop. Although the pathologists were blinded to agent, dose, and exposure interval, the review was intended to provide an overview of the breadth of possible findings, and a venue to describe unique features. Endometrial histology included inactive and normal-appearing cycling endometrium. Overtly premalignant lesions (atypical hyperplasia or EIN) were not seen. In a subset of cases, asymmetry of stromal and epithelial growth resulted in prominent cystically dilated glands with admixed estrogen (mitotic) and progestin (secretory) epithelial effects of a type not encountered in contemporary clinical practice. The variety of endometrial appearances suggested that findings might differ by agent and dose over time according to relationships that must be specified for each agent. The constellation of changes seen in those endometria with cystically dilated glands is so novel that new terminology and diagnostic criteria are required for pathologists to recognize them. The panel has designated these changes as PRM-associated endometrial changes (PAEC). Additional follow-up studies will be needed to fully define their natural history and relationship to specific agents and administration regimens.
\end{abstract}

Modern Pathology (2008) 21, 591-598; doi:10.1038/modpathol.2008.19; published online 1 February 2008

Keywords: progesterone receptor modulators; endometrium; histology; pathology

Progesterone receptor modulators (PRMs) are synthetic compounds that interact with the progesterone receptor to inhibit or stimulate a downstream hormonal response. Those with progesterone antagonist activity have potential application in contraception and treatment of uterine leiomyomata and endometriosis. ${ }^{1}$ Some agents, such as mifepristone (RU486), are already approved in the United States for single use application. New clinical

Correspondence: Dr GL Mutter, MD, Division of Women's and Perinatal Pathology, Department of Pathology, Brigham and Women's Hospital, 75 Francis Street, Boston, MA 02115, USA.

E-mail: gmutter@rics.bwh.harvard.edu

Received 24 September 2007; accepted 3 January 2008; published online 1 February 2008 applications of mifepristone, and other PRMs such as asoprisnil (J867), CDB-2914, and onapristone, are under development. Specifically, efficacy and safety of longer term administration for management of uterine bleeding, endometriosis, and uterine leiomyomata (fibroids) is a subject of active study by the pharmaceutical industry. Over 600,000 hysterectomies are performed annually in the United States, and of these over half (38.6\% leiomyomata, $17.9 \%$ endometriosis or adenomyosis) are initiated for treatment of conditions potentially amenable to medical therapy by PRMs. ${ }^{2}$ The benefit of nonsurgical treatment is especially high for women of reproductive age in whom maintenance of fertility is of paramount importance. If successful, this could result in development of effective and practical 
pharmacologic alternatives to surgical therapy for these diseases.

Assessment of the effects of PRM on the endometrium is of primary interest in understanding the mechanisms of PRM action and ensuring long-term safety. Selective antagonism of the progesterone receptor in premenopausal women who are actively elaborating estrogens is of some concern, as protracted estrogenic stimulation of the endometrium has been associated with a dose- and durationdependent 2- to 10-fold increased risk for endometrial cancer. ${ }^{3-5}$ Under physiological conditions, it is the intermittent elaboration of progesterone during the luteal phase of the menstrual cycle that opposes the otherwise mitogenic effects of estrogens. The pharmacological effects of PRMs are, however, not purely antagonistic, nor is their effect restricted to the progesterone receptor itself. Some PRMs exert anti-estrogenic effects, either through partial progesterone receptor agonist activity, ${ }^{6,7}$ or, exert these effects independently of the progesterone receptor itself, that is by upregulation of the androgen receptor response. ${ }^{8,9}$ These PRM effects are evident as dose-dependent suppression of estrogen-induced mitotic activity, ${ }^{10-12}$ and appearance of glandular secretory changes. ${ }^{7}$

Assessment of PRM induced changes in the endometrium by pathologists is not only a prerequisite step to determine suitability for widespread clinical use, but also indicates how community pathologists are likely to respond to endometrial biopsies from women on these agents. Pathologists simply do not have a historical or established community experience with long-term PRM administration to use as a basis for interpretation. Access to material is generally confined to individuals involved in ongoing clinical trials, members of the parent pharmaceutical companies, a few researchers, and regulatory agencies. Now that the histological features referable to PRM administration are starting to emerge,,$^{7,12-15}$ there are unanswered questions of how they relate, if at all, to known diagnostic entities such as endometrial hyperplasia.

On 7-8 April 2006, in Bethesda, MD, the Center for Population Research at the National Institute of Child Health and Human Development convened a workshop on the subject of 'Progesterone Receptor Modulators and the Endometrium.' Members of industry, the National Institutes of Health, the Food and Drug Administration, and researchers working on PRMs were invited to present and discuss PRM effects on the endometrium from multiple perspectives. The goal was to bring together many of the interested parties at this critical juncture between development and potential clinical application of PRMs, both to share their own experiences and discuss the outstanding issues. Some general aspects of the meeting, including detailed review of the pharmacokinetics and endocrine effects of PRMs, have been published elsewhere. ${ }^{16}$
The organizers realized that the histology of PRM-exposed endometria had presented interpretive difficulties to pathologists, particularly because some of the findings were imperfect matches for established diagnostic entities. A specially convened panel of experienced pathologists was assembled prior to the meeting and provided with a set of endometrial biopsy specimens from women taking PRMs as part of completed or ongoing clinical trials. The pathologists were asked to interactively examine and discuss the specimens and present their consensus observations at the April meeting. Because the pathologists were blinded to the agents and regimens, several of which were represented in the study set, this is not a report of results from a single trial or even a systematic comparison between drugs. Rather, it distills the diagnostic issues faced by reviewing pathologists serving on a PRM clinical trial or a pathologist seeing these changes for the first time in practice. This paper reports the findings of the pathology panel and concludes with their recommendations for future pathological assessments.

\section{Materials and methods}

Eighty-four hematoxylin- and eosin-stained histological sections of endometrial biopsies or curettings were supplied by pharmaceutical companies and study pathologists for review by a panel of seven pathologists with experience in gynecologic pathology. All slides were obtained from women currently taking one of the following four PRMs as part of clinical trials: mifepristone (Danco, New York, NY, USA; University of Rochester, Rochester, NY, USA; and University of Edinburgh, Edinburgh, UK), CDB$2914^{17}$ (HRA Pharma, Paris, France); JNJ-17072341 (Johnson and Johnson Pharmaceutical Research and Development, LLC, Raritan, NJ, USA); or asoprisnil (TAP Pharmaceutical Products Inc., Lake Forest, IL, USA).

Slides were anonymously coded and presented by the chair of the pathology panel ( $G$ Mutter) at a multi-headed microscopy session, to the complete pathology panel (C Bergeron, L Deligdisch, A Ferenczy, M Glant, M Merino, ARW Williams). Discussion continued throughout the microscopy session, allowing detailed and directed examination of all features of each case by the entire panel. At the end of microscopic review of individual cases, the pathology panel convened to develop a consensus summary of their findings, which was presented on 7 April 2007 at the 'Progesterone Receptor Modulators and the Endometrium' workshop held in Bethesda, MD. ${ }^{16}$ The current report summarizes and illustrates these pathology panel consensus conclusions.

At the request of the pharmaceutical companies that supplied materials for this study, all pathologists remained blinded throughout to the agents and 
application regimens of anonymized subjects. Although the materials submitted included representative examples across multiple doses as available to the companies at the time, the method of series assembly was not random and excluded untreated controls. Specific findings as represented in the study set are not necessarily proportional to that seen in the source clinical trial groups. Exclusive of the current effort, systematic compilation and publication of the source clinical trials results will be done by the companies elsewhere. This study was not intended to anticipate or reproduce that effort, but rather describe the experience of an expert pathologist reviewer in assigning diagnostic or descriptive labels to the material.

\section{Results}

The histological appearance of study samples varied greatly, from normal physiological cycling endometrium to easily classified benign pathologies and artifacts, to novel constellations of changes that defy classification using existing diagnostic criteria and terminologies. There were several cases of stromal changes resembling a progestin effect, including normal-appearing secretory endometrium (Figure 1a) and secretorily exhausted glands with pseudodecidualized stroma (Figure 1b). Additionally, there were a few examples of benign and otherwise common conditions, such as benign endometrial polyps and endometritis. These did not appear any different from those encountered in women from the general population. There were some proliferative endometria with cystically dilated glands that were indistinguishable from a disordered proliferative, or anovulatory, endometrium. There were no overtly premalignant lesions, such as atypical endometrial hyperplasia or endometrial intraepithelial neoplasia, within the material, nor were there any carcinomas.

There was a class of abnormal endometria that did not fit into any existing standard diagnostic group, and demonstrated non-physiological combinations of individual histological features that are not seen in normal cycling endometrium. The common theme among novel endometria in the series was coexistence within a single sample of individual features usually attributed separately to changes caused by hormonal depletion, or stimulation by estrogens or progesterone. Inactive (Figure 2a) or apoptotic (Figure 2b) endometrial glands appear in the context of a completely intact stroma, without any evidence of recent stromal breakdown. Ciliated tubal metaplasia, under normal circumstances an estrogen-associated change, was present in otherwise inactive, or even secretory, endometrium. Individual glands demonstrated unusual combinations of mitotic activity with secretory change (Figure 2c) or apoptotic degeneration (Figure 2d).

The appearance of cystically dilated glands was a frequent architectural backdrop for these peculiar admixtures of hormonal effects within the glandular epithelium itself. In its simplest form, scattered cysts were only moderately dilated, much as would be encountered in a disordered proliferative endometrium caused by unopposed estrogens of anovulation (Figure 3a). Instead of the proliferative lining, which this architecture would suggest, the glands were only weakly mitotic or sometimes secretory (Figure 3b). In its most extreme form, extensive confluent cysts were defined by thin flaccid walls resembling cystic or senile atrophy such as seen in some postmenopausal women (Figure 3c). Glands in those cases were not atrophic, but had peculiar combinations of apoptotic and mitotically active epithelial cells (Figure 3d). Other examples of
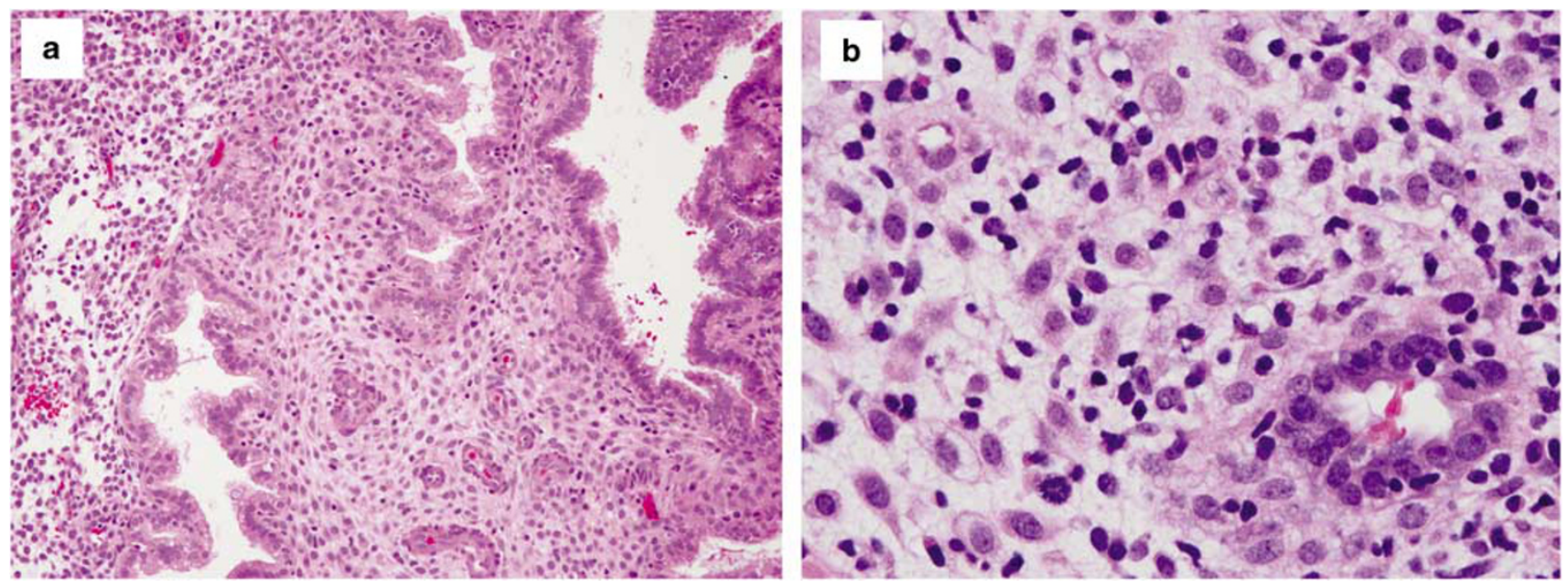

Figure 1 Stromal progestin effect. Rarely, progestational stromal changes were seen as a predecidual response in otherwise normalappearing secretory endometrium (a), or a pseudodecidual response and secretorily exhausted glands resembling that seen with pharmacologic therapy with progestins (b). The majority of patients on PRMs do not show these features. 

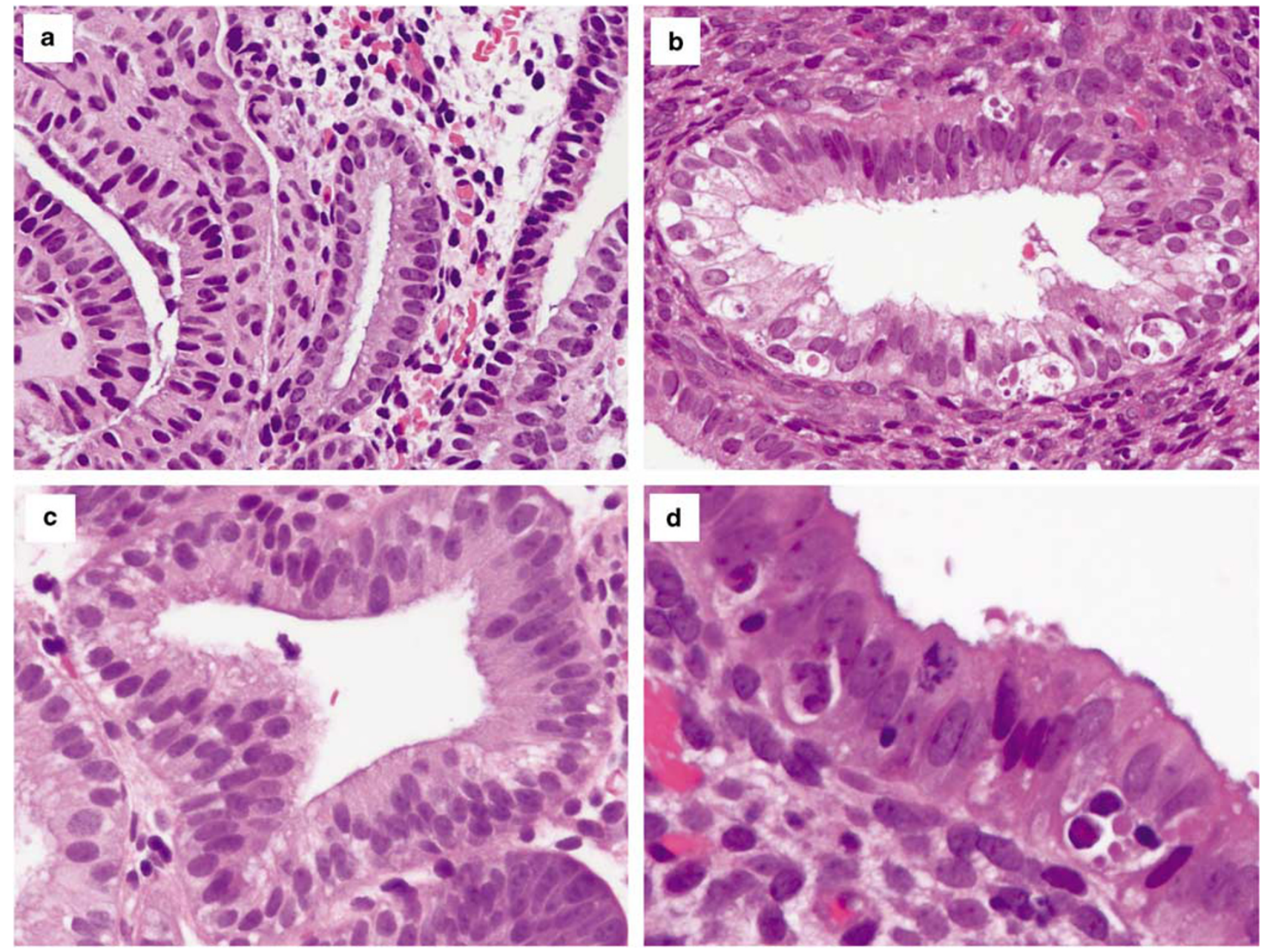

Figure 2 Admixed hormonal effects in endometrial glands. Individual cases contained constellations of features that are not normally seen in premenopausal women. This included inactive (a) or extensively apoptotic (b) amitotic glands with intact stroma. Mitotically active glands, when present, tended to be combined with secretory change (c), or apoptotic degeneration (d).

multicystic endometrium contained interspersed glands with tubal metaplasia or secretory change. Although these large-scale architectures bear superficial resemblance to established diagnostic categories of disordered proliferative, non-atypical cystic hyperplastic, and cystic atrophic endometrium, the panel members agreed that the discordant epithelial cytology was inconsistent with those labels. Typically, many cystic structures were filled with secretory material.

Within the stroma, odd vascular patterns were seen. Prominent, but delicate, anastomosing capillary networks in a 'chicken wire' pattern were widespread in some cases (Figure 4a). Other specimens had thick-walled vessels such as those seen in polyps, but these were widely disseminated throughout the endometrial compartment rather than being deployed in a localized manner, and the affected fragments lacked the altered stroma of polyps (Figure 4b). Stromal blood vessels were occasionally ectatic, with prominent endothelial cells (Figure 4c), but never demonstrated fibrin thrombi of the sort that are seen in unopposed estrogen states.

\section{Discussion}

A novel pattern of PRM-associated changes was seen, demonstrating dyssynchronous growth between glands and stroma, and unusual epithelial types not seen in the normal menstrual cycle of a premenopausal woman. Large-scale architecture was characterized by interspersed cysts throughout the endometrial compartment, composed of glands demonstrating non-physiological combinations of inactivity, mitoses, secretory change, and apoptosis. Individual glands simultaneously displayed mixed features of estrogen and progesterone stimulation. Although fairly distinctive, when they did occur, these changes were present in only a subset of all exposed patients, and perhaps only for some agents at particular doses. Because the histological changes were so variable, it was not possible to use 

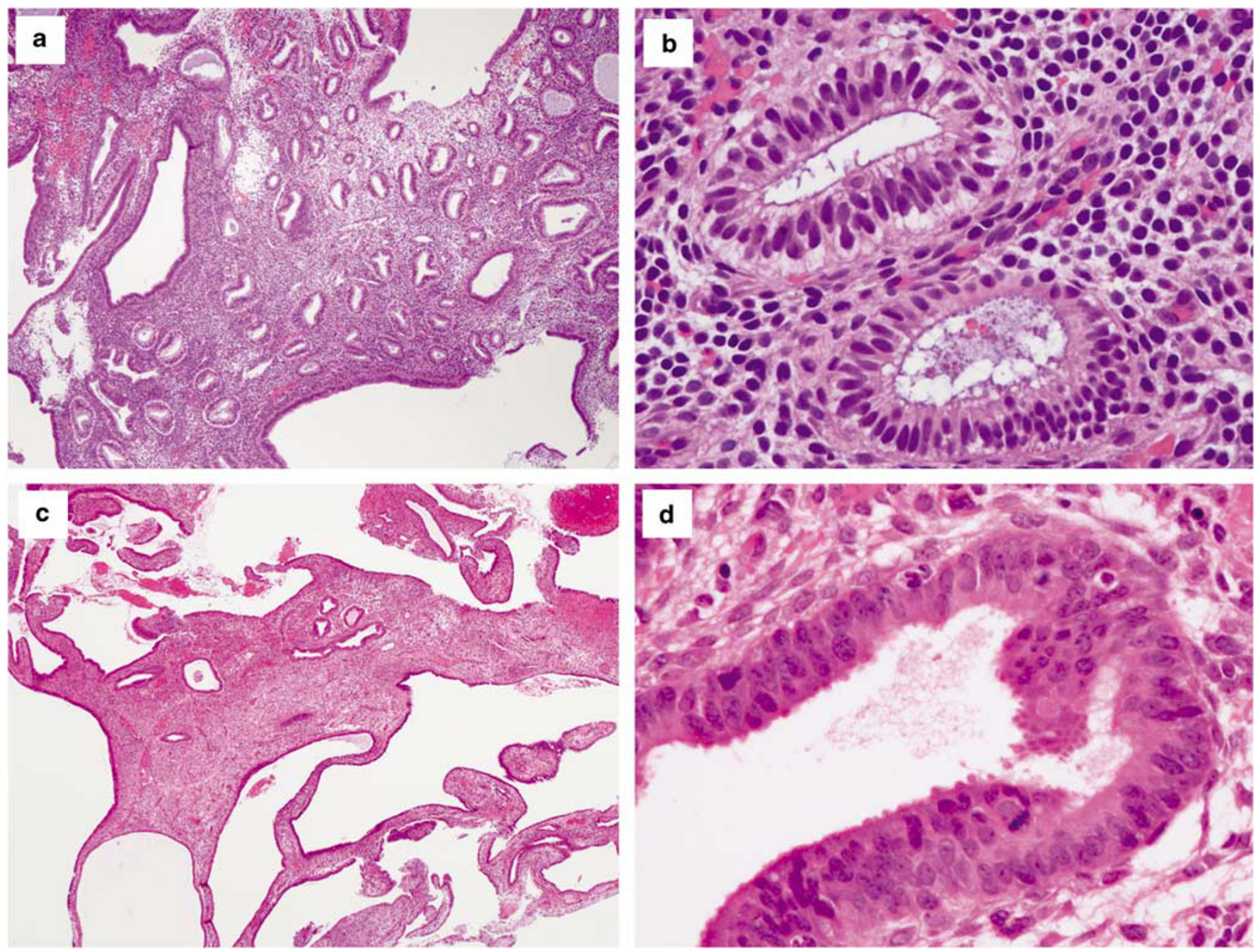

Figure 3 PRM Associated Endometrial Changes (PAEC). A unique presentation among PRM-treated women is a multicystic endometrium in which glands are lined by an incongruous epithelial type. One pattern had moderately dilated glands, much as would be encountered in a disordered proliferative endometrium (a), but which on closer examination instead demonstrate extensive secretory change devoid of accompanying stromal pre decidualization (b). Another pattern at low magnification resembles cystic, or senile, atrophy (c), but is made up of weakly mitotically active glands punctuated by apoptotic bodies (d).

endometrial histology alone to consistently identify patients taking PRMs.

Similar non-physiological endometrial changes have been published previously in limited reports available for PRM-treated women. Cyst formation and mixed progestin agonist/antagonist effects have been described for the PRMs asoprisnil ${ }^{7}$ and VA2914. ${ }^{18}$ This 'non-physiological secretory effect' had an appearance that differed by dose, including decreasing mitotic figure count as the dose of the agent increased. A similar effect has been described with mifepristone, in which higher doses induce androgen receptors, a change with known antiestrogen effects. ${ }^{8,9}$ Changes in agent dose potentially modify the endometrial appearance by varying affinities for multiple steroid hormone receptors, and for each receptor class, a dose dependentbalance of antagonist with agonist activity.

Our impression was that the physiological separation between estrogen- and progesterone-induced changes, in which progesterone downregulation of estrogen receptors prevents co-stimulation of both pathways, was perturbed in the PRM-exposed patients. In the normal menstrual cycle, the follicular phase is dominated by the effects of estrogen, a hormone known to increase mitotic activity. Beginning at the time of ovulation, and extending through the later, luteal, phase, progesterone elaborated by the corpus luteum overrides the effects of estrogen to suppress glandular mitoses and induce secretory change and stromal pre-decidualization. In the premenstrual window, rapid declines in both hormones are responsible for apoptotic-mediated stromal breakdown and secondary degeneration of glands. Thus, under normal conditions, these changes occur in a sequentially orchestrated manner in which estrogen- and progesterone-driven changes are separated by time and occur in a minimally overlapping manner.

Stromal compaction and thick-walled vessels were observed to a degree, but the thick-walled vessels are clustered and without real stromal 

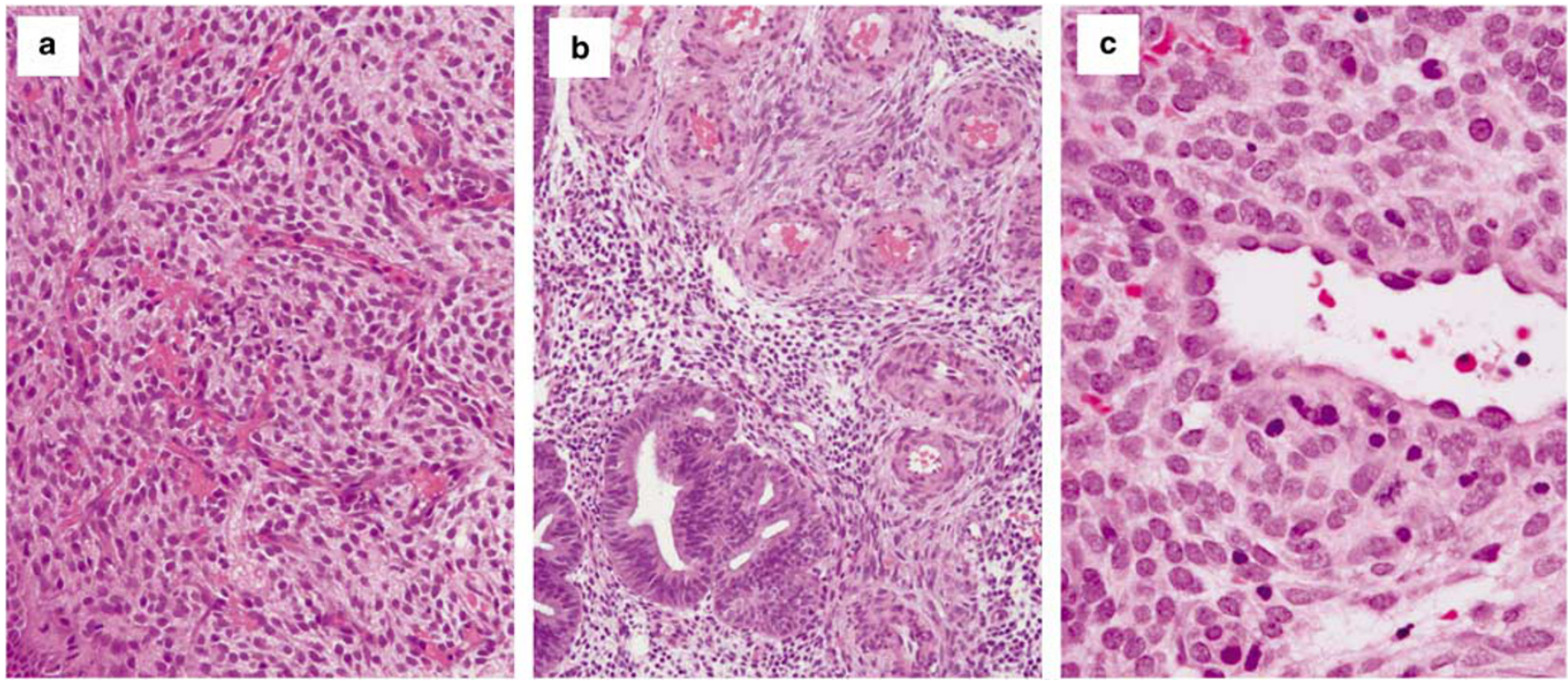

Figure 4 Unusual PRM-associated stromal vascular changes. Prominent, but delicate, anastomosing capillary networks in a 'chicken wire' pattern were widespread in some cases (a). Other specimens had thick-walled vessels such as those seen in polyps, but these were widely disseminated throughout the endometrial compartment rather than being deployed in localized fashion, and the affected fragments lacked the stromal and glandular features of polyps (b). Stromal blood vessels were occasionally ectatic, with prominent endothelial cells (c), but never demonstrated fibrin thrombi of the sort that are seen in unopposed estrogen states.

fibrosis. Similar vessels have been described for the PRM asoprisnil. ${ }^{7}$ The stromal changes seen were unique and differed from those in endometrial polyps. In bland septated cysts, stroma appeared almost fibrous, whereas in other examples nuclei appeared plump with mitotic figures. With a few exceptions, shown in Figure 1, we did not see the kind of stromal change normally associated with progesterone, in particular the stroma did not develop progesterone-specific features to the extent that the epithelium did. In this regard, the glandular and stromal compartments were dyssynchronous, to an extent and direction that was haphazard between the subjects.

The PRM-specific changes described above differ from a pure or classic unopposed estrogen effect in (1) having low or absent mitotic activity; (2) demonstrating varying levels of epithelial secretory change; (3) having occasional stromal pseudodecidual change; and (4) lacking fibrin thrombi and stromal breakdown. They can also be distinguished from the effects of progesterone superimposed upon a pre-existing unopposed estrogen effect, such as is seen in delayed ovulation or progestin-treated anovulation. In that condition, a protracted estrogenic interval progressively increases the density and size of intermittent cysts, which are lined by proliferative epithelium. When delayed ovulation (or low-level progestin administration) occurs, the background cystic architecture remains, but the epithelium develops varying amounts of secretory change and the stroma may demonstrate predecidual change. ${ }^{19}$ These dynamically abnormal menstrual cycles can be envisioned as a trajectory of continuous change in which the histology at any moment is highly dependent on the combination, dose, and timing of hormonal exposure. What offsets the PRM-exposed endometria from these manifestations of an abnormal menstrual cycle is the complete lack of breakdown and fibrin thrombi, and the appearance of estrogen and progestational changes at one point in time rather than in sequence. The PRM-related changes are probably equally dynamic, and it will be important to determine whether there is a defined succession of endometrial changes overtime in PRM-treated women, and how the sequence and composition of observed histologies changes between agents and administration schedules.

The unique PRM-induced changes described above may be referred to as PRM-associated endometrial changes (PAEC).

Novel histological findings pose particular difficulties when pathologists are constrained to application of established diagnostic terms during a clinical study. We have noted superficial resemblance of the irregular cystic architecture of PRM-altered endometria to disordered proliferative, non-atypical cystic hyperplastic and senile cystic atrophic endometrium. The cytologic appearance of the lining epithelium, however, is not quite right for these entities. The panel acknowledges that lesion pathobiology and natural history might not necessarily follow that of these previously described entities, and recommends caution in extrapolation of existing terminologies and their expected clinical outcomes to novel conditions such as these.

It is difficult to judge from this study the longterm outcomes that might follow the endometrial changes seen in women taking PRMs. It will be 
important to determine whether there is a defined succession of endometrial changes time in PRMtreated women, and how the sequence and composition of observed histologies changes between agents and administration schedules. Follow-up data are needed to determine whether the novel changes remain, disappear, or progress to something else. The rarely observed normal appearing, secretory, and stromal pseudodecidual changes were unexpected in women taking progesterone-antagonizing PRMs. It is unclear whether these patients received sub-therapeutic doses, had an idiosyncratic response, or partial progestational agonist activity is capable of mimicking normal histological changes. Notably absent, however, were either discrete or overt premalignant lesions of atypical hyperplasia or EIN.

Even though the constellation of histological elements observed may defy simple classification and thus be difficult to study as a cohesive entity, the individual component features have all been seen before and are amenable to individual analysis. It would be possible, for example, to examine mitotic activity, apoptotic death, or specific stromal changes as single variables irrespective of the context in which they occur. Such a reductionist approach would provide a constrained but comparable benchmark to assess differences across multiple agents and doses.

\section{Recommendations}

The panel in this study concluded with the following recommendations for pathological interpretation of endometrial changes in women taking PRMs:

(1) Endometrial histological findings among women taking PRMs are sufficiently diverse that we can expect they will differ by agent, dose, administration schedule, and patient context.

(2) It is not clear whether novel changes induced by PRMs are in steady state or whether they change dynamically overtime. Multiple time points will be needed to address this question.

(3) Endometrial findings are so novel that new terminology and diagnostic criteria need to be developed. This is a first step to associating long-term clinical outcomes with particular histological appearances, and avoidance of misclassification by pathologists in a routine diagnostic setting.

(4) We suggest use of the term PAEC to describe the endometrial changes from non-physiological hormonal stimulation that are manifest as admixed estrogen and progestin epithelial effects in the context of stromal-epithelial dyssynchrony. This specifies the underlying mechanism as PRM-induced and appropriately describes the perturbed process of tissue morphogenesis.

(5) When endometrial findings are novel, long-term clinical outcomes will need to be measured directly, rather than extrapolated from an imperfect or poor fit to prior experience.

\section{Acknowledgements}

The pathology panel would like to thank the following organizations that generously provided slides from their clinical studies for the pathology review: mifepristone (Danco, New York, NY, University of Rochester, Rochester, NY, USA, University of Edinburgh, Edinburgh, UK); CDB-2914 (HRA Pharma, Paris, France); JNJ-17072341 (Johnson and Johnson Research and Development, LLC, Raritan, NJ, USA); and asoprisnil (TAP Pharmaceutical Products Inc., Lake Forest, IL, USA). Additional meeting support was provided by the Division of Cancer Treatment and Diagnosis, the Division of Cancer Treatment, and the Office of Women's Health of the National Cancer Institute and the National Institutes of Health (NIH) Office of Research on Women's Health.

\section{Disclosure/conflict of interest}

The following authors have no disclosures to make: CB, LD, AF, MM, DB. The following authors have received compensation (from the following companies) for consulting services unrelated to production of this manuscript: GM (ReprosTherapeutics, The Woodlands, TX, USA), AW (Bayer Schering Pharma AG, Berlin, Germany; TAP Pharmaceutical Products Inc., Lake Forest, IL, USA; HRA Pharma, Paris, France), and MG (TAP Pharmaceutical, Lake Forest, IL, USA; Eli Lilly and Company, Indianapolis, IN, USA; Wyeth Pharmaceuticals, Madison, NJ, USA).

\section{References}

1 Chabbert-Buffet N, Meduri G, Bouchard P, et al. Selective progesterone receptor modulators and progesterone antagonists: mechanisms of action and clinical applications. Hum Reprod Update 2005;11: 293-307.

2 Keshavarz H, Hillis S, Kieke B, et al. Hysterectomy Surveillance-United States, 1994-1999. Morb Mortal Wkly Rep 2002;51:1-8.

3 Parazzini F, La Vecchia C, Bocciolone L, et al. The epidemiology of endometrial cancer. Gynecol Oncol 1991;41:1-16.

4 Shapiro S, Kelly JP, Rosenberg L, et al. Risk of localized and widespread endometrial cancer in relation to recent and discontinued use of conjugated estrogens. N Engl J Med 1985;313:969-972.

5 Zeleniuch-Jacquotte A, Akhmedkhanov A, Kato I, et al. Postmenopausal endogenous oestrogens and risk of endometrial cancer: results of a prospective study. Br J Cancer 2001;84:975-981.

6 Schubert G, Elger W, Kaufmann G, et al. Discovery, chemistry, and reproductive pharmacology of asoprisnil and related 11beta-benzaldoxime substituted 
selective progesterone receptor modulators (SPRMs). Semin Reprod Med 2005;23:58-73.

7 Williams AR, Critchley HO, Osei J, et al. The effects of the selective progesterone receptor modulator asoprisnil on the morphology of uterine tissues after 3 months treatment in patients with symptomatic uterine leiomyomata. Hum Reprod 2007;22:1696-1704.

8 Slayden OD, Nayak NR, Burton KA, et al. Progesterone antagonists increase androgen receptor expression in the rhesus macaque and human endometrium. J Clin Endocrinol Metab 2001;86:2668-2679.

9 Narvekar N, Cameron S, Critchley HO, et al. Low-dose mifepristone inhibits endometrial proliferation and upregulates androgen receptor. J Clin Endocrinol Metab 2004;89:2491-2497.

10 Murphy AA, Zhou MH, Malkapuram S, et al. RU486induced growth inhibition of human endometrial cells. Fertil Steril 2000;74:1014-1019.

11 Baird DT, Brown A, Cheng LN, et al. Mifepristone: a novel estrogen-free daily contraceptive pill. Steroids 2003;68:1099-1105.

12 Cameron ST, Critchley HO, Thong KJ, et al. Effects of daily low dose mifepristone on endometrial maturation and proliferation. Hum Reprod 1996;11: 2518-2526.

13 Baird DT, Brown A, Critchley HO, et al. Effect of longterm treatment with low-dose mifepristone on the endometrium. Hum Reprod 2003;18:61-68.
14 Brown A, Cheng L, Lin S, et al. Daily low-dose mifepristone has contraceptive potential by suppressing ovulation and menstruation: a doubleblind randomized control trial of 2 and $5 \mathrm{mg}$ per day for 120 days. J Clin Endocrinol Metab 2002;87: 63-70.

15 Eisinger SH, Bonfiglio T, Fiscella K, et al. Twelvemonth safety and efficacy of low-dose mifepristone for uterine myomas. J Minim Invasive Gynecol 2005;12: 227-233.

16 Horne FM, Blithe D. Progesterone receptor modulators and the endometrium: changes and consequences. Hum Reprod Update 2007;13:567-580.

17 Blithe DL, Nieman LK, Blye RP, et al. Development of the selective progesterone receptor modulator CDB-2914 for clinical indications. Steroids 2003;68: 1013-1017.

18 Chabbert-Buffet N, Pintiaux-Kairis A, Bouchard P. Effects of the progesterone receptor modulator VA2914 in a continuous low dose on the hypothalamic-pituitary-ovarian axis and endometrium in normal women: a prospective, randomized, placebo controlled trial. J Clin Endocrinol Metab 2007;92: 3582-3589.

19 Mutter GL, Zaino RJ, Baak JPA, et al. The benign endometrial hyperplasia sequence and endometrial intraepithelial neoplasia. Int J Gynecol Pathol 2007;26: 103-114. 